\title{
Feasibility of ASsisTed WARfarin Dosing by Clinical Pharmacy Support Assistants (FAST-WARD Study)
}

\author{
Damen Man and Vincent H Mabasa
}

\begin{abstract}
Background: Pharmacy-managed warfarin dosing has been established at Burnaby Hospital, in Burnaby, British Columbia, for over 10 years. With increases in the number and acuity of patients enrolled, it has become challenging to maintain a successful anticoagulation program. The clinical pharmacy support assistant (CPSA) program was initiated to support the provision of clinical pharmacy services. At the time of the study, Burnaby Hospital had 2 CPSAs. It was anticipated that the pharmacy-managed inpatient warfarin dosing service might benefit from support through the CPSA program to maintain a consistent level of delivery.

Objective: To examine the feasibility of CPSAs supporting the pharmacy-managed inpatient warfarin dosing service at Burnaby Hospital. Feasibility was based on 4 key parameters: knowledge base, accuracy of data collection, dosage recommendations, and time spent on the process.
\end{abstract}

Methods: An observational, prospective pilot study was conducted over 3 months. The CPSAs were given appropriate education and training, and their performance was assessed to determine the feasibility of CPSAassisted warfarin dosing. The CPSAs had to achieve a priori target scores for each of the 4 parameters in order for CPSA-assisted warfarin dosing to be considered feasible.

Results: After the didactic sections, both CPSAs answered all review questions correctly. The accuracy of data collection (based on 60 patient encounters) was $98.3 \%$. The warfarin regimens recommended by the CPSAs were similar to those recommended by the clinical pharmacists, with doses differing by a mean of $0.46 \mathrm{mg}$. For $39(65 \%)$ of the 60 patient encounters, the dosing recommendations of CPSAs and clinical pharmacists were identical. The average time spent per patient encounter was $10.5 \mathrm{~min}$.

Conclusion: With appropriate training and education, it is feasible for CPSAs to support the pharmacy-managed inpatient warfarin dosing program at Burnaby Hospital.

Keywords: warfarin, clinical pharmacy support assistant, anticoagulation, pharmacy practice

Can J Hosp Pharm. 2014;67(3):220-5

\section{RÉSUMÉ}

Contexte : Un programme d'ajustement posologique de la warfarine géré par le pharmacien est en place à l'hôpital Burnaby depuis plus de 10 ans. Comme le nombre de patients inscrits au programme s'accroit et que la gravité des cas augmente, il est maintenant difficile pour le programme d'anticoagulothérapie de conserver le même niveau de performance. Le programme d'assistant au soutien de la pharmacie clinique (ASPC) a été mis en place dans le but de soutenir la prestation de services de pharmacie clinique. Au moment de l'étude, l'hôpital Burnaby avait deux ASPC. Le service d'ajustement par le pharmacien de la posologie de la warfarine des patients hospitalisés pourrait bénéficier du soutien du programme d'ASPC afin de conserver un niveau de prestation uniforme.

Objectif : Étudier la possibilité pour les ASPC de soutenir le service d'ajustement par le pharmacien de la posologie de la warfarine des patients hospitalisés offert par l'hôpital Burnaby. La faisabilité était fondée sur quatre paramètres clés : la base de connaissances, l'exactitude de la collecte de données, les recommandations posologiques et le temps alloué au processus.

Méthodes : Une étude pilote prospective observationnelle a été menée sur une période de trois mois. Les ASPC ont obtenu un enseignement et une formation adéquate et, suite à cela, leur travail a été évalué dans le but de déterminer si une aide à l'ajustement posologique de la warfarine est possible. Les ASPC devaient atteindre des scores prédéfinis a priori pour chacun des quatre paramètres pour que leur aide à l'ajustement posologique de la warfarine soit considérée comme faisable.

Résultats : Après avoir participé aux volets didactiques, les deux ASPC ont répondu correctement à l'ensemble des questions d'évaluation. Lexactitude de la collecte de données (fondée sur 60 rencontres avec des patients) était de 98,3\%. Les schémas posologiques de warfarine recommandés par les assistants étaient semblables à ceux recommandés par les pharmaciens cliniciens et variaient en moyenne de $0,46 \mathrm{mg}$. Pour 39 (65\%) des 60 rencontres avec des patients, les recommandations posologiques des assistants étaient identiques à celles des pharmaciens cliniciens. Chaque rencontre durait en moyenne 10,5 minutes.

Conclusion : À l'aide d'une formation et d'un enseignement adéquats, il est donc possible pour les ASPC de soutenir le programme d'ajustement par le pharmacien de la posologie de la warfarine des patients hospitalisés offert par l'hôpital Burnaby.

Mots clés : warfarine, assistant au soutien de la pharmacie clinique, anticoagulothérapie, pratique pharmaceutique

[Traduction par l'éditeur] 


\section{INTRODUCTION}

$\mathrm{W}$

arfarin, a vitamin $\mathrm{K}$ antagonist, has been the mainstay of anticoagulant therapy for various treatments and/or prevention of thromboembolic events. ${ }^{1}$ Pharmacy-managed inpatient anticoagulation therapy is at least equal, and sometimes superior, to physician-managed inpatient anticoagulation therapy. ${ }^{2}$ As such, pharmacy-managed warfarin dosing remains a common day-to-day clinical practice for clinical pharmacists in many institutions.

Burnaby Hospital is a community-based, 310-bed acute care facility located in Burnaby, British Columbia. Pharmacymanaged warfarin dosing has been established at Burnaby Hospital for over 10 years. Pharmacists have prescribing authority to monitor and manage patients who are receiving warfarin therapy. This process involves gathering clinically relevant information; deciding on appropriate warfarin dosages while patients are in hospital; discontinuing heparin products when a patient's international normalized ratio (INR) is within the therapeutic range; monitoring for efficacy and safety; and providing counselling and recommendations to patients, family members, and other health care professionals. A continuous quality improvement evaluation of pharmacistmanaged warfarin therapy at Burnaby Hospital showed that this approach was not associated with increases in major and minor bleeding relative to physician-managed warfarin therapy. ${ }^{3}$ As the number and acuity of patients enrolled in the program increases, maintenance of a successful anticoagulation program becomes challenging, especially when no additional resources are available to allocate to the service.

The clinical pharmacy support assistant (CPSA) program was initiated to support the provision of clinical pharmacy services at Burnaby Hospital. At the time this article was prepared, in late 2013, Burnaby Hospital had 2 CPSAs whose daily activities included obtaining, organizing, and distributing patient profiles, related material, and supporting documentation for review by clinical pharmacists; assisting with the retrieval of drug information; printing reports; providing discharge counselling; and participating in various clinical projects. It was anticipated that the pharmacy-managed inpatient warfarin dosing service might benefit from support through the CPSA program to maintain a consistent level of service.

The management of warfarin therapy by pharmacy technicians and assistants is not a new concept, but to date has been reported only from the outpatient setting. ${ }^{4-6}$ The hypothesis was therefore that CPSA-supported, pharmacy-managed warfarin dosing would be feasible if the CPSAs received appropriate education, training, and tools and underwent appropriate quality assessment. The objective of this study was to examine the feasibility of CPSAs supporting the pharmacymanaged inpatient warfarin dosing service at Burnaby Hospital. Feasibility was based on 4 key parameters: knowledge base, accuracy of data collection, dosage recommendations, and time spent on the process. The hospital's 2 CPSAs were trained in this program.

\section{METHODS}

Patients enrolled in the Burnaby Hospital pharmacistmanaged inpatient warfarin dosing service (for standardized dosing protocol, see Appendix 1, available online at www.cjhponline.ca/index.php/cjhp/issue/view/102/showToc) between June and August 2011 were included in the study. Patients were excluded if the main indication for warfarin therapy was a need for valve replacement, because of different INR targets. Patients with concomitant medications and those who experienced drug interactions were not excluded, as these are important factors that play a role in making warfarin dosing recommendations in real-life cases.

For this observational, prospective pilot project, a clinical pharmacist reviewed every warfarin recommendation written by a CPSA. The investigators consulted with the Fraser Health Research Ethics Board, which indicated that the study did not require research ethics board approval.

The feasibility of CPSA-assisted warfarin dosing was assessed through 4 key parameters that were considered crucial to patient safety and to the logistic feasibility of this novel practice. The 4 key parameters were knowledge and understanding of anticoagulation, accuracy of data collection, appropriateness of recommended dose, and time spent per patient encounter. The CPSAs had to achieve predefined, a priori target scores for each of the 4 parameters in order for CPSA-assisted warfarin dosing to be considered feasible.

\section{Knowledge Base}

The CPSAs participated in 4 didactic sessions on the basics of anticoagulation therapy. The topics covered included disease pathophysiology, warfarin pharmacology, methods and resources for data collection, strategies for making dosage recommendations, and guided real-patient scenarios (Figure 1). Knowledge was tested with a total of 16 multiple-choice questions reflecting the material presented, subdivided by topic (see Appendix 2, available online at www.cjhp-online.ca/ index.php/cjhp/issue/view/102/showToc). The CPSAs had to achieve a score of $90 \%$ or greater for questions pertinent to each session before beginning the next session. 


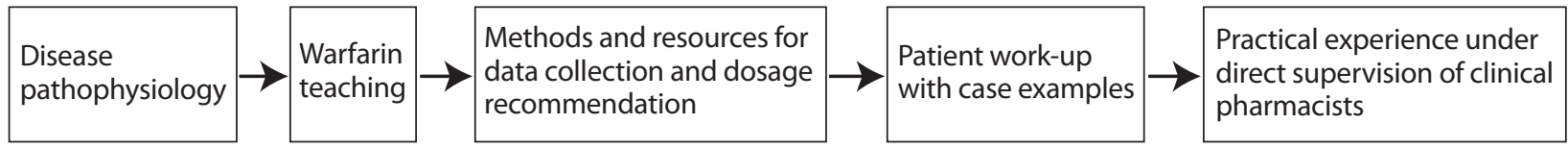

Figure 1. Training curriculum for clinical pharmacy support assistants. The first 4 components represent the didactic sessions described in the text.

Following successful completion of the knowledge base component of the project, the CPSAs were assigned patients for assessment and follow-up of warfarin therapy. For these patients, the CPSAs had to complete the entire work-up process, including data collection, monitoring of efficacy and safety, screening for drug-drug interactions, and dosage recommendations, as described in the sections below. The CPSAs discussed recommended dosages for these patients with the supervising pharmacist.

\section{Accuracy of Data Collection}

The CPSAs used a standardized data collection form (Figure 2) to collect the patient information required for proper assessment of warfarin therapy, including appropriate laboratory data (red blood cell, white blood cell, and platelet counts; INR), patient safety data (bleeding and bruising status), and efficacy of therapy (if patient had previously been receiving warfarin). The CPSAs interviewed the assigned patients and discussed patients' status with nurses. They noted any changes in medication on the data collection form and entered the new drugs into the Micromedex Drug Interaction Database ${ }^{7}$ to check for potential drug-drug interactions. The CPSAs were expected to take such interactions into account when making their dosage recommendations. All data collection forms were audited by the investigators, and accuracy of $95 \%$ or higher was required, where accuracy was defined as the number of correct entries that the investigators detected on the data collection forms (Figure 2).

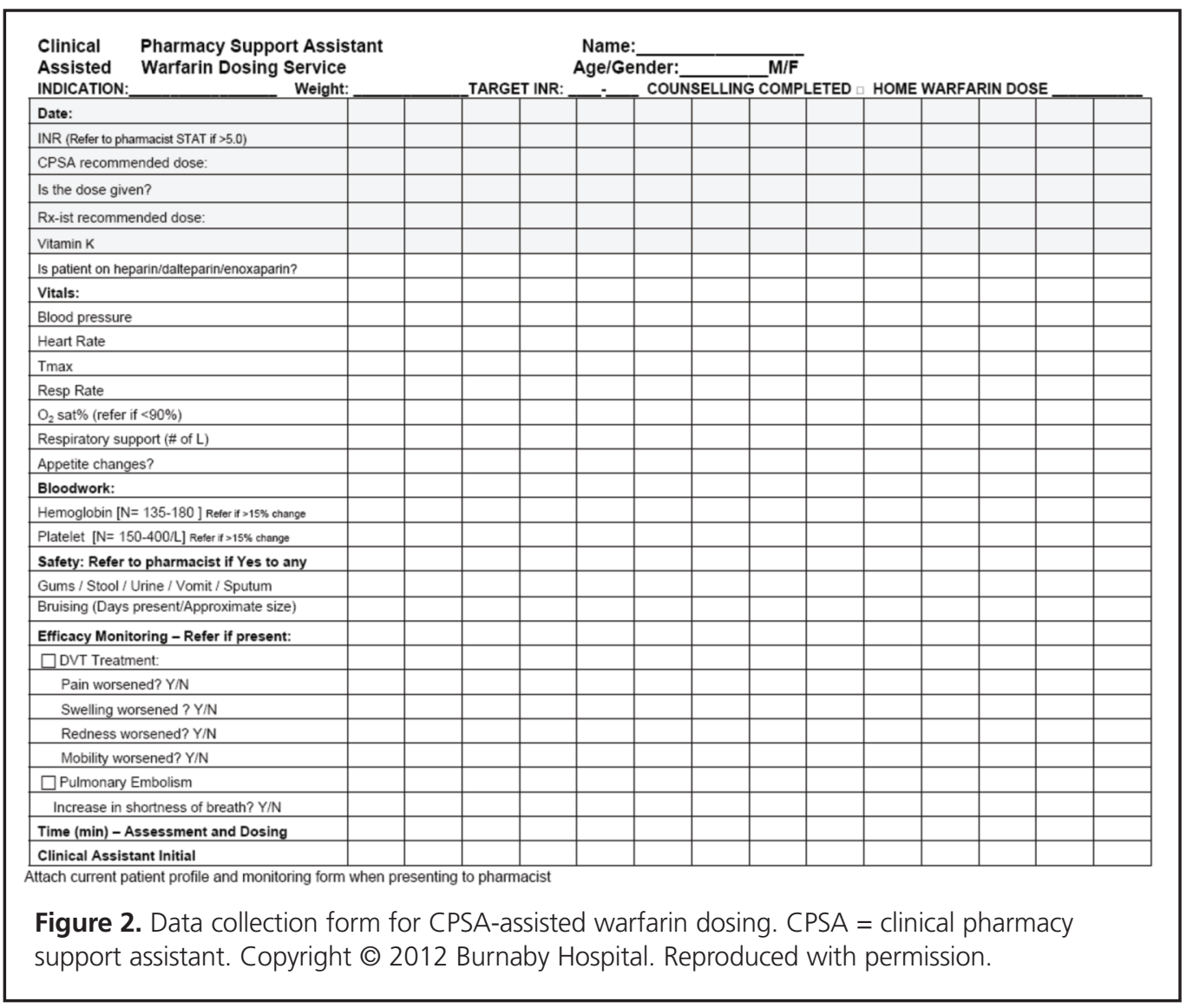




\section{Dosage Recommendations}

Once patient-specific data had been collected, the CPSA was required to make a warfarin dosage recommendation. This recommendation could be based on the existing warfarin protocol (see Appendix 1) or could reflect what the CPSA deemed appropriate for the patient. Each recommendation was then compared against the recommendation of a clinical pharmacist, who evaluated the patient independently. The dosage recommendations were compared in both absolute and relative terms. It was expected that the dosage recommendations made by the CPSAs would differ to some extent from those made by the pharmacists; however, it was determined that the absolute variance in dose should be within $0.5 \mathrm{mg}$ (the smallest available dose increment for warfarin) for each recommendation.

\section{Time Spent on the Process}

Each patient encounter was timed, starting when the CPSA began the patient's work-up. The recorded time included obtaining pertinent laboratory data from the hospital informatics system and patients' charts, interviewing the patient as required, preparing the dose recommendation, and concisely reporting the information gathered to the clinical pharmacist who was looking after the patient. It was determined that the entire process should be completed within $15 \mathrm{~min}$ to be considered cost-neutral relative to the usual time spent by a pharmacist. This cost calculation was based on the average time spent by pharmacists to complete the task ( $8 \mathrm{~min})$ and the respective salaries of pharmacists and CPSAs (pharmacist salary approximately 2 times greater than CPSA salary).

The study was based on a convenience sample of patient encounters over the period June to August 2011. Patient encounters were defined in terms of the number of warfarin dose recommendations made by the CPSAs, including data collection and reporting of pertinent information to clinical pharmacists.

\section{RESULTS}

After the 4 didactic sessions, both of the CPSAs correctly answered all 16 multiple-choice review questions.

Sixty patient encounters took place during the study period, and data collection was completed with no patients lost to follow up. The investigators determined that there was only 1 error in data collection over the entire sample of 60 patient encounters; the CPSAs' accuracy in collecting patient-specific information was therefore $98 \%(59 / 60)$.
Of the 60 warfarin dosing recommendations arising from the patient encounters, 39 (65\%) were identical between the CPSA and the pharmacist. For the remainder, 15 (25\%) of the CPSA doses were lower and $6(10 \%)$ were higher than those recommended by the clinical pharmacists (Figure 3 ). The mean absolute difference in dose was $0.46 \mathrm{mg}$, with a maximum absolute difference of $3 \mathrm{mg}$ for one patient encounter.

The average time spent for each patient encounter was $10.5 \mathrm{~min}$ (standard deviation $3.1 \mathrm{~min}$ ).

\section{DISCUSSION}

This study demonstrated that it is feasible for CPSAs to support the pharmacy-managed inpatient warfarin dosing program at Burnaby Hospital, after receiving appropriate training and education. To the authors' knowledge, this is the first study to consider the utilization of CPSAs to support an inpatient warfarin dosing program and adds to the body of evidence for how CPSAs can support clinical pharmacy services. The results of this study are important for the department as it seeks ways to support the pharmacy-managed inpatient warfarin dosing service and maintain consistent service to a growing number of patients enrolled in the

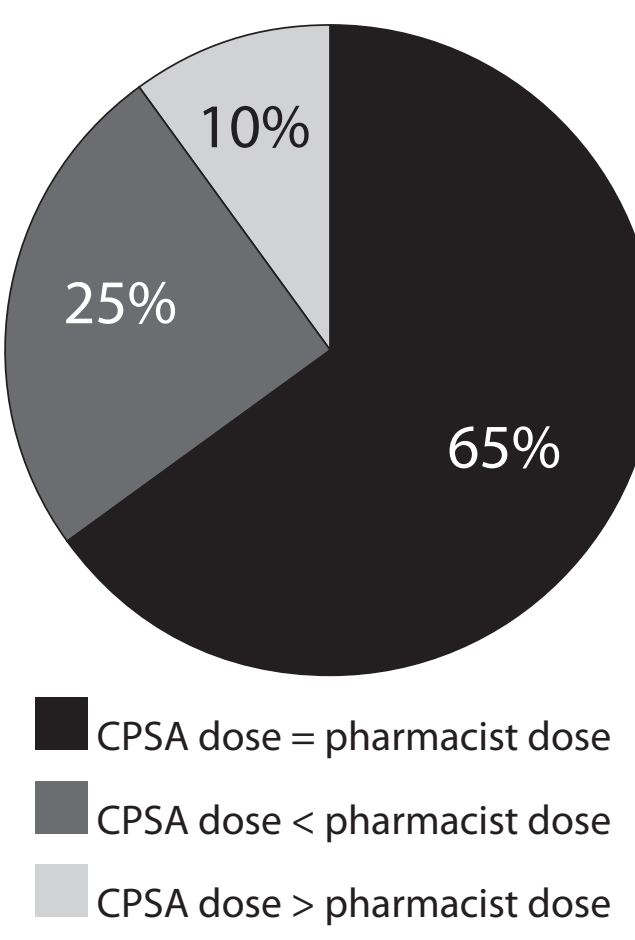

Figure 3. Differences in dosing recommendations between clinical pharmacy support assistants (CPSAs) and clinical pharmacists for 60 patient encounters. 
program. The expanded role of CPSAs may help to further advance the profession of pharmacy technicians and may help to improve the working relationship between pharmacy technicians and clinical pharmacists.

It is important that CPSAs be aware of and understand the details of what is involved in each type of clinical service they support. This is especially true for the pharmacy-managed warfarin dosing service at Burnaby Hospital. The enhanced knowledge that the CPSAs received during the didactic sessions allowed them to perform beyond their usual technical duties and also allowed them to understand the importance of the information that they gathered. For example, recognition of the potentially numerous drug-drug interactions involving warfarin allowed the CPSAs to understand why INR values may change significantly over time and why the clinical pharmacist should be alerted to such a change.

Accuracy of data collection was a key indicator in determining feasibility. The CPSAs demonstrated $98 \%$ accuracy in data collection. The single error in data collection (over the entire sample of 60 patient encounters) did not directly affect the dosage recommended for the patient. The importance of the data collection process and the potential consequences of errors were reviewed and emphasized with the CPSAs.

The CPSAs' understanding of the principles of anticoagulation therapy was more importantly reflected in the dosages they recommended and the monitoring they provided. From this study, it appears that patient safety would not have been compromised if clinical pharmacists had depended on the CPSAs' reports and agreed with their recommendations for warfarin dosages. The CPSAs' dose recommendations were very similar to those of the clinical pharmacists, even in terms of accounting for drug-drug interactions, and for over $60 \%$ of patient encounters, the dosage recommendations were identical. At first glance, this value may seem lower than desirable; however, given that the mean dose difference was $0.46 \mathrm{mg}$ and given that there may be differences even between clinical pharmacists, this is actually a significant and positive result. Another interesting observation was that the CPSAs were in general more conservative in their dosing practices than the clinical pharmacists. It would be interesting to follow this trend over time to see if their dosing practices change as they become more experienced.

In assessing the logistics and feasibility of this novel practice, time was an important factor. Although the average time required by CPSAs during this study was longer than that required by clinical pharmacists (10.5 min versus $8 \mathrm{~min}$ ), the CPSAs' time per encounter declined over the course of the study. The reported average reflects the actual time spent but does not reflect the possibility of increasing efficiency. Notably, for the latter half of the sample, most patient encounters were completed in about $8 \mathrm{~min}$, which more accurately reflects the typical amount of time that trained and experienced CPSAs require to complete a patient encounter. Future studies should assess the cost-effectiveness of this practice and the amount of clinical pharmacist time that can be saved, relative to the amount of CPSA time devoted to the warfarin dosing service.

The study had some limitations. First, the sample size was rather small because of limited ability of the CPSAs to take on additional work. Second, the sample of patient encounters was selected by the clinical pharmacists on duty and might have been subject to selection bias. This type of bias could be minimized in future studies through a randomization process. Third, only patients who were receiving warfarin therapy for atrial fibrillation and treatment or secondary prophylaxis of a venous thromboembolic event were assigned to a CPSA. This may limit the types of patients that CPSAs can monitor in the future. However, a recent drug utilization evaluation study at Burnaby Hospital showed that patients taking warfarin for other indications represented less than $5 \%$ of the patient population (unpublished data). Finally, the study did not assess the quantitative benefits of this type of support program to the pharmacy department. It will be important to determine whether the program is cost-effective and whether there are any effects on patient outcomes, such as number of days to reach therapeutic INR after warfarin is started, number of days in supratherapeutic INR range, and rates of bleeding.

This study has laid a foundation for future investigations involving CPSAs assisting with warfarin dosing and anticoagulation management. It also suggests a new way of increasing technicians' responsibility and job satisfaction.

\section{CONCLUSION}

With appropriate training and education, it is feasible for CPSAs to support the pharmacy-managed warfarin dosing program for inpatients at Burnaby Hospital.

\section{References}

1. Ansell J, Hirsh J, Hylek E, Jacobson A, Crowther M, Palareti G; American College of Chest Physicians. Pharmacology and management of the vitamin K antagonists: American College of Chest Physicians EvidenceBased Clinical Practice Guidelines (8th edition). Chest. 2008;133(6 Suppl):160S-198S.

2. Donovan JL, Drake JA, Whitaker P, Tran MT. Pharmacy-managed anticoagulation: assessment of in-hospital efficacy and evaluation of financial impact and community acceptance. J Thromb Thrombolysis. 2006;22(1):23-30. 
3. Cheng L. Continuous quality improvement evaluation of pharmacist managed warfarin therapy at Burnaby Hospital [poster presentation]. Presented at Burnaby Hospital grand rounds; 2008 Jul 16.

4. Thomas M, Cross S, Nagra M, Bullock A. Focus on technicians: empowering technicians to work in extended clinical roles. Hosp Pharm. 2008; $15: 145-6$

5. Hobson J. Focus on technicians: make a technician an active part of the team. Hosp Pharm. 2003;10:283-5.

6. Keresztes JM. Role of pharmacy technicians in the development of clinical pharmacy. Ann Pharmacother. 2006;40(11):2015-9.

7. Micromedex Drug Interaction Database. Truven Health Analytics; (c) 2012-2014. Available from: www.micromedexsolutions.com/micromedex2/ librarian/ND_T/evidencexpert/ND_PR/evidencexpert/CS/B147DE/ND_ AppProduct/evidencexpert/DUPLICATIONSHIELDSYNC/1E8110/ ND_PG/evidencexpert/ND_B/evidencexpert/ND_P/evidencexpert/ PFActionId/evidencexpert.FindDrugInteractions (subscription required to access content)
Damen Man, BSc(Pharm), ACPR, PharmD, was, at the time this study was performed, a Staff Pharmacist at Burnaby Hospital, Burnaby, British Columbia. He is now a Clinical Pharmacy Specialist-Intensive Care, Abbotsford Regional Hospital, Abbotsford, British Columbia.

Vincent H Mabasa, BSc(Pharm), ACPR, PharmD, is Clinical Coordinator, Burnaby Hospital, Burnaby, British Columbia.

Competing interests: None declared.

Address correspondence to:

Dr Damen Man

Pharmacy Department

Abbotsford Regional Hospital

32900 Marshall Road

Abbotsford BC V2S OC2

e-mail: damen.man@fraserhealth.ca

Acknowledgements

We thank Alan Wei for his contributions to the early stages of this project. We also thank Marsha Fajardo, Karen Hertslet, and Patty Tarbet, who worked as clinical pharmacy support assistants (CPSAs) at Burnaby Hospital over the course of the project.

\section{CISADL 2013}

\section{Order your copy of the 2013 Canadian Investigational \& Special Access Drug List today!}

\section{Please use the CSHP order form found online at CSHP.ca to place your order.}

The CISADL is a compilation of drugs that are currently not marketed in Canada and may have Investigational or Special Access status with Health Canada. This list is not endorsed by Health Canada's Special Access Program. For precise information about the regulatory status of a drug, contact the Special Access Program directly. Entries to the list are contributed to by several Drug Information Centers across Canada and by Canadian drug manufacturers. In cases where non-marketed drugs do not appear on the list, foreign references should be consulted. The content of the list includes generic name and strength/concentration, dosage form, pharmacology/therapeutic use, trade and code name, source (manufacturer/distributor), and comments. Available in English only.

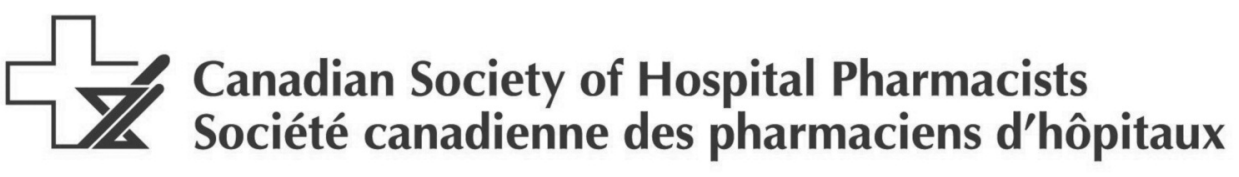

